\title{
Successful Surgery for Scoliosis Supported by Pulmonary Rehabilitation in a Duchenne Muscular Dystrophy Patient With Forced Vital Capacity Below 10\%
}

Jang Woo Lee, $\mathrm{MD}^{1}$, Yu Hui Won, $\mathrm{MD}^{2}$, Won Ah Choi, $\mathrm{MD}^{1}$, Soon Kyu Lee, $\mathrm{MD}^{1}$, Seong Woong Kang, $\mathrm{MD}^{1}$

${ }^{1}$ Department of Rehabilitation Medicine and Rehabilitation, Institute of Neuromuscular Disease, Yonsei University College of Medicine, Seoul; ${ }^{2}$ Department of Rehabilitation Medicine, Ewha Womans University School of Medine, Seoul, Korea

Low vital capacity is a risk factor for scoliosis correction operation in Duchenne muscular dystrophy (DMD) patients, but pulmonary rehabilitation, including noninvasive intermittent positive pressure ventilator application, air stacking exercise, and assisted coughing technique, reduces the pulmonary complications and perioperative mortality risk. In this case, the patient's preoperative forced vital capacity (FVC) was $8.6 \%$ of normal predicted value in sitting position and $9.4 \%$ in supine position. He started pulmonary rehabilitation before the operation and continued right after the operation. Scoliosis correction operation was successful without any pulmonary complications, and his discomfort in sitting position was improved. If pulmonary rehabilitative support is provided properly, FVC below $10 \%$ of normal predicted value is not a contraindication of scoliosis correction operation in DMD patients.

Keywords Scoliosis, Low vital capacity, Duchenne muscular dystrophy, Pulmonary rehabilitation

\section{INTRODUCTION}

In patients with Duchenne muscular dystrophy (DMD), respiratory failure is common due to weaknesses of the respiratory muscles, and it is one of the most common causes of mortality [1]. However, pulmonary rehabilita-

Received June 15, 2012; Accepted August 11, 2012

Corresponding author: Seong Woong Kang

Department of Rehabilitation Medicine and Rehabilitation, Gangnam Severance Hospital, 211 Eonju-ro, Gangnam-gu, Seoul 135-720, Korea Tel: +82-2-2019-3492, Fax: +82-2-3463-7585, E-mail: kswoong@yuhs.ac

(c) This is an open-access article distributed under the terms of the Creative Commons Attribution Non-Commercial License (http://creativecommons. org/licenses/by-nc/3.0) which permits unrestricted noncommercial use, distribution, and reproduction in any medium, provided the original work is properly cited.

Copyright $\odot 2013$ by Korean Academy of Rehabilitation Medicine tion, such as ventilatory support by home ventilator and air-stacking exercise, not only reduces respiratory complications, but also prolongs the mean survival time [2]. In addition, the correction of scoliosis has been increasingly performed to maintain lung volume capacity and sitting posture, even if the patient cannot perform the activities of daily living [3]. In the past, low forced vital capacity (FVC) was thought to increase complications in major operations [1], so scoliosis correction was performed with limitations. However, in the past 10 years, cases of successful operations were reported by Takaso et al. [3] and Gill et al. [4] in patients with neuromuscular disease with low FVC below $30 \%$ of predictive value. There is no report of successful operation in patients with very low FVC with $10 \%$ of predictive value in previous 
studies. This is the first report of successful scoliosis correction with no respiratory complications achieved by active and comprehensive pulmonary rehabilitation in a DMD patient with very low FVC with $10 \%$ of predictive value.

\section{CASE REPORT}

Herein, we report a 19-year-old male patient who suffered from gait disturbance since the age of 2 years when he often fell and had difficulty with climbing stairs. At the age of 7 , he was diagnosed as DMD by muscle biopsy, and follow-up in the out-patient department of Gangnam Severance Hospital was stopped at his parents' will. He had not walked since 10 years of age and had been suffering from symptoms of hypercapnea, such as sleeping during the daytime and headache upon awakening from sleep. In July 2010, when he turned 18 years old, he was visited by the home visiting service of Gangnam Severance Hospital, and his partial pressure of end-tidal carbon dioxide $\left(\mathrm{EtCO}_{2}\right)$ was above $50 \mathrm{mmHg}$. He was admitted to the Department of Physical Medicine and Rehabilitation at Gangnam Severance Hospital on August 7, 2010. In the arterial blood gas analysis, partial pressure of carbon dioxide $\left(\mathrm{PaCO}_{2}\right)$ was $49.9 \mathrm{mmHg}$ and partial pressure of oxygen $\left(\mathrm{PaO}_{2}\right)$ was $91.8 \mathrm{mmHg}$. In the evaluation of pulmonary function, FVC in sitting position was 460 $\mathrm{mL}$ (8.6\% of predictive value), FVC in supine position was $500 \mathrm{~mL}$ ( $9.4 \%$ of predictive value), unassisted peak cough flow (UPCF) was $80 \mathrm{~L} / \mathrm{min}$, and maximum insufflation capacity (MIC) was $1,390 \mathrm{~mL}$. In the overnight transcutaneous monitoring of partial pressure of carbon dioxide (SenTec Digital Monitor System with V-Sign Sensor; SenTec AG, Therwil, Switzerland), retention of carbon dioxide was noticed, as the maximal $\mathrm{PaCO}_{2}$ was $76.6 \mathrm{mmHg}$ and the mean $\mathrm{PaCO}_{2}$ was $64.6 \mathrm{mmHg}$. He received training for noninvasive intermittent positive pressure ventilation (NIPPV), which mode is assist-controlled ventilation (ACV). During application of NIPPV, the overnight monitoring showed a reduction in carbon dioxide retention, as the maximal $\mathrm{PaCO}_{2}$ was $46.1 \mathrm{mmHg}$ and the mean $\mathrm{PaCO}_{2}$ was $40.6 \mathrm{mmHg}$. He was prescribed a home ventilator, and the ventilator settings were the mode of ACV, tidal volume of $1,150 \mathrm{~mL}$, and respiration rate of 16 times per minute. Based on the daytime monitoring of $\mathrm{PaCO}_{2}$, after 6 -hour discontinuance of home ventilator, hypercapnea had redeveloped; he was therefore educated to apply the home ventilator while sleeping and additionally in the daytime. Furthermore, he was educated to exercise airstacking using a manual resuscitator bag and home ventilator, more than 3 times a day. After application of home ventilator, the symptoms of hypercapnea were resolved.

After being discharged, the patient had no complaints related to respiratory failure, but he suffered from difficulty sitting even if he leaned back, due to severe scoliosis; Cobb's angle was $58.1^{\circ}$. He was considered high risk for scoliosis operation due to a very low FVC of less than $10 \%$ of predictive value. Although he was thought to be safe from respiratory complications after the scoliosis correction, he was trained on a home ventilator for 6 months to maintain proper ventilator states via NIPPV immediately after extubation. At the Department of Orthopedic Surgery, he underwent surgeries for anterior fixation from the fifth lumbar vertebra to the first sacral vertebra on February 10, 2011, and for posterior fixation from the second thoracic vertebra to the first sacral vertebra on February 22, 2011. During the operations, his vital signs were stable. From the time of admission, noninva-
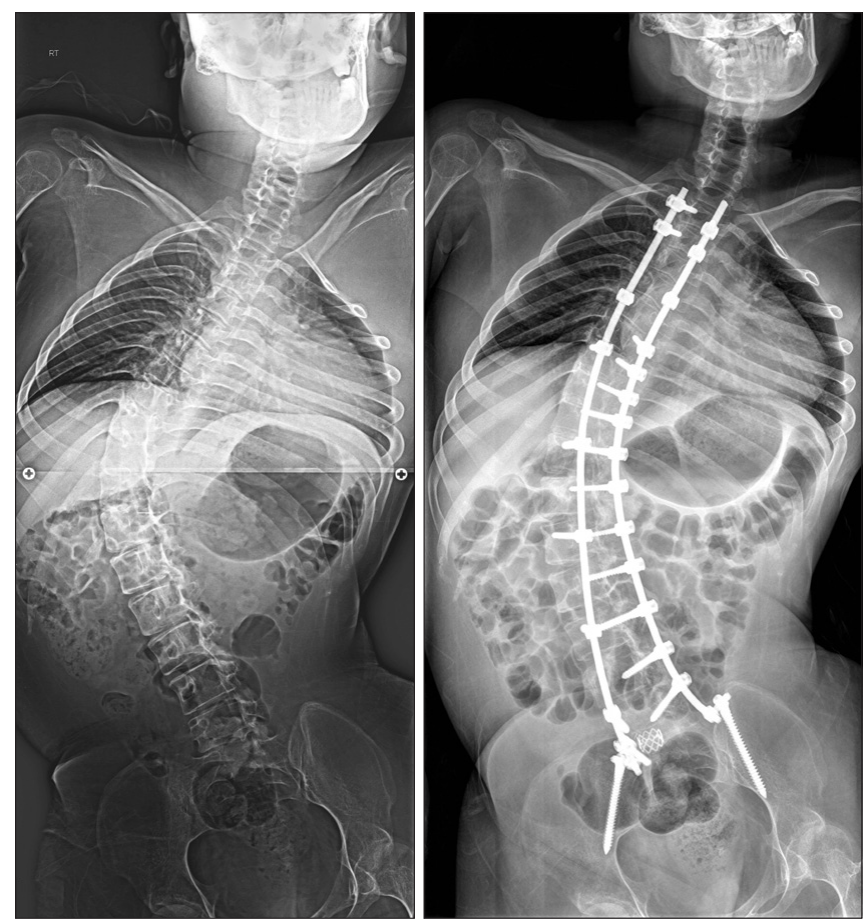

Fig. 1. Preoperative and postoperative whole spine X-rays show improved scoliosis. Cobb's angle on preoperative $\mathrm{X}$-ray was $58.1^{\circ}$ and the postoperative angle was $48.5^{\circ}$. 
Table 1. Evaluation of preoperative and postoperative pulmonary function

\begin{tabular}{lcccc}
\hline & \multicolumn{4}{c}{ Date of evaluation } \\
\cline { 2 - 5 } & $\mathbf{2 0 1 0 - 0 8 - 0 9}$ & $\mathbf{2 0 1 1 - 0 2 - 0 8}$ & $\begin{array}{c}\mathbf{2 0 1 1 - 0 4 - 1 4} \\
\text { (POD \#51) }\end{array}$ & $\begin{array}{c}\mathbf{2 0 1 1 - 1 2 - 1 9} \\
\mathbf{( P O D} \# \mathbf{3 0 0})\end{array}$ \\
\hline VCsit $(\mathrm{mL})$ & $460(8.6)$ & $510(9.6)$ & $590(11.1)$ & $520(9.8)$ \\
VCsupine $(\mathrm{mL})$ & $500(9.3)$ & $530(9.9)$ & $580(10.9)$ & $660(12.5)$ \\
\hline MIC $(\mathrm{mL})$ & $1,390(26.1)$ & $1,010(19.0)$ & $1,740(32.8)$ & $1,880(35.6)$ \\
\hline UPCF $(\mathrm{L} / \mathrm{min})$ & 80 & 60 & 110 & 90 \\
APCF $(\mathrm{L} / \mathrm{min})$ & 220 & 200 & 240 & 290 \\
MIPsit $\left(\mathrm{cmH}_{2} \mathrm{O}\right)$ & 10 & 15 & 13 & 20 \\
MIPsupine $\left(\mathrm{cmH}_{2} \mathrm{O}\right)$ & 11 & 13 & 19 & 19 \\
MEPsit $\left(\mathrm{cmH}_{2} \mathrm{O}\right)$ & 12 & 13 & 12 & 8 \\
\hline MEPsupine $\left(\mathrm{cmH}_{2} \mathrm{O}\right)$ & 10 & 17 & 12 & 10 \\
\hline
\end{tabular}

Values are presented as number (\%).

VCsit, vital capacity at sitting position; VCsupine, vital capacity at supine position; MIC, maximal insufflation capacity; UPCF, unassisted peak cough flow; APCF, assisted peak cough flow; MIP, maximum inspiratory pressure; MEP, maximum expiratory pressure; POD, postoperative day.

sive $\mathrm{PaCO}_{2}$ monitoring was conducted during sleep. After serial surgeries, he was transferred to the intensive care unit with maintenance of the intubation tube; expectoration of sputum and air-stacking were implemented by mechanical insufflator-exsufflator (MIE). The first day after the operation, NIPPV via home ventilator was applied right after intubation tube removal. Upon confirmation that there were no complications and hypercapnea (the maximal and mean $\mathrm{PaCO}_{2}$ were 25 and $20.5 \mathrm{mmHg}$, respectively), he was transferred to the general ward.

At 8 days after the surgery, intravenous antibiotics were injected due to infection of the surgical wound. On April 9, 2011, 46 days after the operation, all stitches were removed. Cobb's angle was changed from $58.1^{\circ}$ preoperatively to $48.5^{\circ}$ postoperatively (Fig. 1). He still can sit only with a supporting back, but subjective comfort was improved by the reduction of the inclination in the sitting position. At postoperative day 51 and 300, pulmonary function was reevaluated. FVC, assisted peak cough flow (APCF), and MIC increased; MIC was particularly improved from $1,010 \mathrm{~mL}$ preoperatively to $1,880 \mathrm{~mL}$ postoperatively, although UPCF showed no significant change (Table 1).

\section{DISCUSSION}

Scoliosis in DMD patients is a common complication, and is aggravated at the age of loss of ambulatory func- tion. Severe scoliosis commonly induces many problems, such as imbalance of the sitting position, decreased cardiopulmonary function, musculoskeletal pain, pressure ulcers, and so on [5]. Also, high pressure on the thoracic cage and abdomen makes respiration and expectoration difficult, and mechanical ventilatory support and assistive coughing methods are needed. It is difficult to assess the cardiac function via electrocardiography or echocardiography, because of poor positioning and approach for diagnostic devices. Scoliosis operation is beneficial for not only correcting Cobb's angle, but also improving the quality of life, including the sitting balance, activities of daily living, back pain, and cardiopulmonary function [6]. However, decline of cardiopulmonary function increases the risk of complications with a major operation, such as pneumonia, pneumothorax, heart failure, and prolongation of invasive ventilatory support [7]. Rawlins et al. [1] reported that $19 \%$ of patients with neuromuscular disease and FVC below $40 \%$ of predictive value suffer respiratory complications after surgical scoliosis correction, and in the past, in patients with FVC below $30 \%$ of predictive value, scoliosis operation was contraindicated [8]. However, in studies in the past 10 years, there was no significant difference in risk even if the FVC was below $30 \%$ of predictive value $[3,4]$, and in patients with low FVC, surgical correction of scoliosis was received relatively safely [4].

In patients with neuromuscular disease, if respiratory 
muscles are weakening, the tidal volume decreases and the respiratory rate increases to compensate for the reduced tidal volume. Accordingly, fatigue of the respiratory muscles induces retention of carbon dioxide in the body. The patient in this case had a very low FVC below $10 \%$ of predictive value. Because of hypercapnea, he needed to be assisted by NIPPV, and normal ventilatory states were maintained during sleep. After the application of NIPPV for more than 6 months, it is possible to change from intubation to NIPPV immediately after a major operation without difficulty.

After surgery, management of sputum is very important for the prevention of atelectasis and pneumonia. In patients with neuromuscular disease, decreased ability to expectorate is a major cause of respiratory complications after operation. The patient in this case had UPCF of 60 $\mathrm{L} / \mathrm{min}$, which was lower than $160 \mathrm{~L} / \mathrm{min}$, the minimal value for toileting of sputum by himself. However, APCF was $200 \mathrm{~L} / \mathrm{min}$ with support of air-stacking and manual abdominal thrust, and additional application of MIE made it easy to remove airway secretions and advance the schedule of extubation.

Scoliosis in neuromuscular disease can decrease FVC by an additional $12 \%-16 \%$ of predictive value [9]. This patient had increased MIC after surgical correction of scoliosis, even though there was minimal change in FVC. MIC is measured by additional manual insufflation by a manual resuscitator bag in the states of maximal inspiration using one's inspiratory muscles [10]. It is thought that the increased MIC is caused by steady training with air-stacking exercise using a manual resuscitator bag and home ventilator, as well as by a more secured space for expansion of the lung after scoliosis operation. Also, increased APCF resulted from increased MIC and ease for manual assist through the improvement in the sitting balance (Table 1).

Based on this case, despite very low FVC below $10 \%$ of normal predictive value, surgical scoliosis correction can be performed successfully without operation-related complications in DMD patients if pulmonary rehabilitative support, such as NIPPV, assist coughing methods, air-stacking, and application of MIE, is provided.

\section{CONFLICT OF INTEREST}

No potential conflict of interest relevant to this article was reported.

\section{REFERENCES}

1. Rawlins BA, Winter RB, Lonstein JE, Denis F, Kubic $\mathrm{PT}$, Wheeler $\mathrm{WB}$, et al. Reconstructive spine surgery in pediatric patients with major loss in vital capacity. J Pediatr Orthop 1996;16:284-92.

2. Kang SW. Pulmonary rehabilitation in patients with neuromuscular disease. Yonsei Med J 2006;47:307-14.

3. Takaso M, Nakazawa T, Imura T, Okada T, Fukushima $\mathrm{K}$, Ueno M, et al. Surgical management of severe scoliosis with high risk pulmonary dysfunction in Duchenne muscular dystrophy: patient function, quality of life and satisfaction. Int Orthop 2010;34:695-702.

4. Gill I, Eagle M, Mehta JS, Gibson MJ, Bushby K, Bullock R. Correction of neuromuscular scoliosis in patients with preexisting respiratory failure. Spine (Phila Pa 1976) 2006;31:2478-83.

5. Cheuk DK, Wong V, Wraige E, Baxter P, Cole A, N'Diaye T, et al. Surgery for scoliosis in Duchenne muscular dystrophy. Cochrane Database Syst Rev 2007;(1):CD005375.

6. Larsson EL, Aaro SI, Normelli HC, Oberg BE. Longterm follow-up of functioning after spinal surgery in patients with neuromuscular scoliosis. Spine (Phila Pa 1976) 2005;30:2145-52.

7. Master DL, Son-Hing JP, Poe-Kochert C, Armstrong DG, Thompson GH. Risk factors for major complications after surgery for neuromuscular scoliosis. Spine (Phila Pa 1976) 2011;36:564-71.

8. Sussman MD. Advantage of early spinal stabilization and fusion in patients with Duchenne muscular dystrophy. J Pediatr Orthop 1984;4:532-7.

9. Yamashita T, Kanaya K, Yokogushi K, Ishikawa Y, Minami R. Correlation between progression of spinal deformity and pulmonary function in Duchenne muscular dystrophy. J Pediatr Orthop 2001;21:113-6.

10. Kang SW, Cho DH, Lee SC, Moon JH, Park YG, Song NK, et al. Clinical implication of air stacking exercise in patients with neuromuscular diseases. J Korean Acad Rehabil Med 2007;31:346-50. 